This is a preprint of a revised manuscript submitted to PlosOne

1 Full title: Should we keep some distance from distancing? Regulatory and postregulatory effects of emotion downregulation.

6 Kersten Diers ${ }^{1}$, Denise Dörfel ${ }^{1 *}$, Anne Gärtner ${ }^{1}$, Sabine Schönfeld ${ }^{1}$, Henrik Walter ${ }^{2}$, Alexander 7 Strobel ${ }^{1}$, Burkhard Brocke ${ }^{1}$

8 Scientific articles usually go through a peer-review process. This means that independent 9 researchers evaluate the quality of the work, provide suggestions, and speak for or against the 10 publication. Please note that the present article has not (yet) undergone this standard procedure for scientific publications.

$13 \quad{ }^{1}$ Faculty of Psychology, Technische Universität Dresden, Dresden, Germany

$14{ }^{2}$ Division of Mind and Brain Research, Department of Psychiatry and Psychotherapy, CCM, Charité 15 Universitätsmedizin, Berlin, Germany

$19 *^{*}$ Corresponding author:

20 Email: denise.doerfel@tu-dresden.de (DD 
This is a preprint of a revised manuscript submitted to PlosOne

\section{Abstract}

Emotion regulation is an indispensable part of mental health and adaptive behavior. Research

23 into emotion regulation processes has largely focused on the concurrent effects of volitional emotion

24 regulation. However, there is scarce evidence considering post-regulatory effects with regard to

25 neural mechanisms and emotional experiences. Therefore, we compared concurrent effects of

26 cognitive emotion regulation with effects at different (immediate, short- and long-term) time

27 intervals. In an fMRI study with $\mathrm{N}=46(\mathrm{~N}=30$ at re-exposure) young healthy adults, we compared

28 neuronal responses to negative and neutral pictures while participants had to distance themselves

29 from or to actively permit emotions in response to these pictures. We investigated the temporal

30 dynamics of activation changes related to regulation in cognitive control brain networks as well as in

31 the amygdala during stimulation (concurrent effects, timepoint 1) and post-stimulation (immediate,

32 timepoint 2), as well as during re-exposure with the same pictures after short (10 minutes, timepoint

33 3) and long (1 week, timepoint 4) time intervals. At timepoint 1, negative pictures (versus neutral

34 pictures) elicited a strong response in regions of affective processing, including the amygdala.

35 Distancing (as compared to permit) led to a decrease of this response, and to an increase of activation

36 in the right middle frontal and inferior parietal cortex. We observed an interaction effect of time

37 (stimulation vs. post-stimulation) and regulation (distance vs. permit), indicating a partial reversal of

38 regulation effects during the post-stimulation phase (timepoint 2). Similarly, after 10 minutes

39 (timepoint 3) and after 1 week (timepoint 4), activation in the amygdala was higher during pictures

40 that participants were previously instructed to distance from as compared to permit. These results

41 show that the temporal dynamics are highly variable both within experimental trials and across brain

42 regions. This can even take the form of paradoxical aftereffects at immediate and persistent effects at 43 prolonged time scales. 
This is a preprint of a revised manuscript submitted to PlosOne

\section{Introduction}

Research on emotion regulation is based on the basic tenet that subjective experience and physiological reactions are susceptible to voluntary regulation efforts, and that this constitutes an adaption to complex and changing environments [1]. While there are different strategies of emotion regulation, according to the Process Model of Emotion Regulation [2], they all have in common that they alter the subjective experience of an emotion by means of (cognitive) top-down influences.

The Process Model of Emotion Regulation [2] distinguishes five strategies of emotion regulation according to the timepoint in the emotion generation process at which they are implemented: Situation selection, situation modification, attentional deployment, cognitive change

53 (often referred to as reappraisal), and response modulation. A recent taxonomy [3] builds on this

54 model and further specifies the terminology (to be) used in past and future emotion regulation research. Each strategy comprises different tactics. For instance, situation selection might be achieved by behavioral avoidance, modification by problem solving, attentional deployment via distraction, and response modulation by suppression of the emotional expression. In this study, we focus on cognitive change, or reappraisal, that has been repeatedly reported as adaptive emotion regulation

59 strategy in terms of both (short-term) effectiveness and relation to psychopathology [3-6]. Reappraisal

60 appears early in the emotion generation process (antecedent-focused) and refers to altering the value

61 of the emotion eliciting stimulus. It has been assumed that reappraisal is advantageous because the emotional response has not fully unfolded and the affective value of an event itself is altered [6, 7].

63 Reappraisal can be further divided into different tactics, as well [3]: Reinterpretation, i.e. changing 64 the meaning of a stimulus [8], and distancing (previously often referred to as detachment) [see also

65 our own work, 9, 10]. The meta-analysis by Webb, Miles and Sheeran (4) showed that while 66 reappraisal has been proven effective overall, distancing $\left(d_{+}=0.45\right)$ was more advantageous than 
This is a preprint of a revised manuscript submitted to PlosOne

67 reinterpretation $\left(\mathrm{d}_{+}=0.36\right)$. According to Powers and LaBar (3), distancing can take different forms:

68 Emotion downregulation by taking a perspective a) that is more spatially distant from the stimulus

69 (spatial), b) that is more distant in time (temporal), c) in which the stimulus represents a hypothetical

70 scenario as opposed to reality (hypothetical), or d) by taking an objective perspective (objective).

71 Most (neuroimaging) studies investigating distancing including our own, so far, implemented

72 experimental designs using the objective form: Participants are instructed to take the perspective of a

73 non-involved, objective observer in order to downregulate emotional responses $[9,11]$. Objective

74 distancing has been shown to effectively downregulate negative emotions and activate brain regions

75 implied in cognitive control $[3,4,12]$, offering an effective form of emotion regulation to investigate

76 neural correlates and temporal dynamics of successful emotion regulation.

At a neural level, emotion regulation, including cognitive change and attentional deployment,

is implemented by cortical regions that exercise influence over emotion-generating subcortical regions [13]. Specifically, emotion-generating regions such as the amygdala have been shown to be responsive to both up- and down-regulation; conversely, regions within the dorsal and ventral frontal cortex, the inferior parietal cortex, and the cingulate cortex are regarded as parts of a common cognitive emotion regulation network $[12,14,15]$. These two systems are assumed to be negatively coupled [16, 17], although there have been conflicting findings [18]. Further research has shown that cortical regions are selectively recruited for the regulation of different kinds of stimuli, for example positive vs. negative [19], for different regulation goals such as up- vs. down-regulation [8], and for different of regulation strategies, e.g. distancing, expressive suppression, or distraction [10, 17, 20, 21]. Taken together, this suggests that while a common and potentially indispensable control network of cognitive emotion regulation can be identified, additional structures are selectively recruited only under certain circumstances, leading to highly context-dependent patterns of co-activation of different

90 brain structures. 
This is a preprint of a revised manuscript submitted to PlosOne

The effects of these networks, however, are not consistently stable over time and cannot be

92 fully understood without the consideration of their evolution across multiple time scales. The idea is

93 that regulation processes act differently at distinct temporal stages of the emotion-generating process

94 and even beyond, that is, after the cessation of the stimulus. Most studies so far have focused on

95 concurrent effects of active emotion regulation. However, it has been shown that emotion regulation

96 effects are also observed beyond the active regulation period, that is during the subsequent post-

97 stimulation phase [immediate effects, see for distancing 9]. In detail, the authors showed an

98 immediate increase of amygdala signal once active regulation via distancing had ended. This was

99 described as a rebound, or paradoxical aftereffect. To qualify as such, the signal needs to demonstrate

100 a crossed interaction effect between time and task, that is decreased concurrent amygdala activation

101 during the stimulation (picture presentation) period and increased activation during the immediate

102 post-stimulation period for a comparison of regulation vs no-regulation conditions. This concept has

103 been extended to a number of brain regions and timepoints. Lamke and colleagues [22] observed

104 decreased activation during emotion regulation in the amygdala and in visual cortex regions in the

105 stimulation phase but increased activation in the subsequent post-stimulation phase. Yet, increased

106 activation was observed during both stimulation and post-stimulation period in the dorsolateral

107 prefrontal cortex and other cortical regions, leading to a distinction between reverse and concordant

108 task-rest interactions.

One study [9] demonstrated not only immediate aftereffects, but also effects across an even

110 more extended period of time up to several minutes (short-term effects). Specifically, upon re-

111 encountering previously regulated negative stimuli in a passive viewing task ten minutes later, the

112 authors observed a persisting down-regulation of the amygdala. This re-exposure effect was

113 negatively correlated to the paradoxical aftereffect that was observed immediately after the

114 stimulation phase; i.e., a higher immediate aftereffect was associated with a smaller short-term 
This is a preprint of a revised manuscript submitted to PlosOne

115 regulation effect. A similar study [23] investigated concurrent and long-term effects (1 day) of both

116 emotional up- and down-regulation. After one day, lasting experiential and neural effects were only

117 observed for down-regulation of negative feelings via reinterpretation, but not for other tactics

118 (distraction) or other goals of regulation (up-regulation). Further, the lasting neural effects were

119 confined to prefrontal regions, and were not observed in the amygdala. Independently, it has been

120 shown that regulation effects are also present on long-term time scales of more than one day:

121 Amygdala responses remained attenuated after one week for images that had previously been

122 reappraised [using objective distancing, 24]. This, however, was only observed within the amygdala,

123 but not the frontal cortex, and only happened when the images had been presented and reappraised

124 repeatedly. Another example of long-term effects has been established for episodic memory

125 processes: Down-regulation via distancing during encoding led to prefrontal cortical activation during

126 recognition after one year, whereas lack of regulation during encoding led to activation of rather

127 affective brain regions after the same interval [25].

As systematic investigations of the temporal dimension of distancing including several time intervals are still lacking, the present research aims at replicating and extending previous results on the temporal dynamics of distancing, that is its concurrent, immediate, short- and long-term consequences. We implement a full two-by-two design and compare negative and neutral pictures, achieving a joint maximization of both arousal and valence differences under two task conditions: to

133 distance from (regulation) and to actively permit (no-regulation) all emotions that might arise from 134 negative and neutral pictures. We use multiple timepoints to disentangle the temporal dynamics: 1) a 135 stimulation phase to measure concurrent activation differences between regulation and no-regulation, 136 2) a post-stimulation phase to investigate immediate aftereffects, 3) a re-exposure phase after 10 137 minutes (short-term) as well as 4) a re-exposure phase after one week (long-term) to investigate 138 prolonged aftereffects of distancing. 
This is a preprint of a revised manuscript submitted to PlosOne

We expected that this experimental design would lead to activation increases within the

140 amygdala in response to negative pictures, as compared to neutral ones, and that these activation

141 increases would be subject to modulation by the concurrent condition: During the stimulation phase

142 (timepoint 1), we expected relatively less activation in the amygdala when participants were 143 instructed to distance from negative pictures as compared to permitting all upcoming emotions. This 144 should be accompanied by activation increases within several cortical regions, in particular within 145 the frontal and parietal cortex as an indicator of processes associated with cognitive emotion 146 regulation, more specifically objective distancing. Besides the replication of these expected canonical 147 results, we sought to investigate possible paradoxical aftereffects within the amygdala: We expected 148 that the lower amygdala activation in the distance condition as compared to the permit condition in 149 the stimulation phase reverses in the post-stimulation phase (immediate aftereffect, timepoint 2). We 150 further aimed at characterizing the amygdala response during re-exposure with the same pictures after 151 short (10 minutes, timepoint 3$)$ and long (1 week, timepoint 4) time intervals [9, 22].

\section{Materials and methods}

This study presents data collected within a larger project on neural correlates and individual differences in emotion regulation and its aftereffects (SFB 940 Project A5). Specifically, the project aimed at elucidating the effectiveness and potential costs of volitional emotion regulation. Different cognitive regulation strategies (acceptance, up- and down-regulation) were compared with respect to their behavioral and neural effectiveness, i.e., emotion regulation success, but operationalized along a prolonged time scale. This allowed to examine potential costs of volitional emotion regulation as indexed by paradoxical immediate and delayed regulatory after-effects. Further aims of the overall

160 project were to investigate associations of emotion regulation success with personality traits and 161 genetic polymorphisms. A detailed overview of the project and its subprojects is given in the 
This is a preprint of a revised manuscript submitted to PlosOne

162 Supplementary Materials (Fig S1). Therefore, some of the data reported in this article have been

163 reused in three follow-up studies within the scope of this project: in accordance with the a priori 164 specified analysis plan (http://gepris.dfg.de/gepris/projekt/223659428 and https://tu165 dresden.de/bereichsuebergreifendes/sfb940/research/a-mechanismen/a5), associations with genetic 166 polymorphisms were investigated [26], as well as the relation between emotion regulation and 167 personality [27]. Additionally, associations of emotion regulation success and dispositional emotion 168 regulation with resting-state cortico-limbic connectivity have been analyzed [18]. Results from the 169 present sample on the research questions of this publication have not been reported in any of these 170 publications. We report how we determined our sample size, all data exclusions (if any), all 171 manipulations, and all measures in our study, as recommended by transparency guidelines [28]. All 172 procedures performed in studies involving human participants were in accordance with the ethical 173 standards of the institutional and/or national research committee and with the 1964 Helsinki 174 declaration and its later amendments or comparable ethical standards. The experimental protocol was 175 approved by the ethics committee of the Technische Universität Dresden (EK10012012). Data and 176 materials are provided at the Open Science Framework (https://osf.io/mg5ac/).

\section{Participants}

Sample size was defined based on feasibility considerations. This resulted in a target sample

179 size of over 48 participants. The sample size that we considered feasible to collect, enabled us with

180 the possibility to at least detect medium-sized effects at a significance level of 0.05 and a power of 1810.80 . Forty-eight volunteers (16 male, age range $18-38$ years, age mean $\pm S D=24.6 \pm 4.1$ years) 182 were recruited from the university community. All participants were right-handed and did not report 183 any current or prior neurological or psychiatric illness or treatment. All participants provided written 184 informed consent and received financial compensation. One participant did not complete the 
This is a preprint of a revised manuscript submitted to PlosOne

experiment, and another participant withdrew from the study. MRI data were therefore available for 46 (out of 48) participants. Out of these 46 participants, 30 returned after 1 week for their second MRI measurement and were included in the analyses of the follow-up measurements (re-exposure, timepoint 4), while 10 returned at an earlier or later time (after 6 to 30 days) and were hence not included in the present analysis, and 6 did not return at all for the second session.

\section{Experimental paradigm and procedure}

The study consisted of two sessions, one week apart [for detailed descriptions see 18, 27]. During the first session (60 $\mathrm{min})$, participants performed a preparatory scan $(5 \mathrm{~min})$, four runs of an emotion regulation task (36 $\mathrm{min})$, an anatomical scan $(8 \mathrm{~min})$ and a re-exposure task (10 min). During the second session (25-35 $\mathrm{min})$, participants performed a preparatory scan $(5 \mathrm{~min})$, a resting state measurement $(8 \mathrm{~min})$ and repeated the re-exposure task $(10 \mathrm{~min})$. Additionally, participants were asked to fill in questionnaires with regard to individual differences in emotion regulation and their subjective experience during the fMRI measurement as well as provided a blood sample, which are not focused on in the present publication [for a detailed description see 26, 27].

\section{Emotion regulation task (timepoints 1 and 2)}

During the emotion regulation task, participants were asked to either permit the emotions arising in response to a set of negative and neutral pictures, or to down-regulate them by means of objective distancing. During the "permit" condition, participants were asked to take a close look at the picture and permit any emotions that might arise as a result. They were encouraged to imagine that they were immediately witnessing the depicted situation, and instructed not to voluntarily intensify their emotions, to re-interpret the situation, or to distract themselves. During the "distance" condition, they were asked to "take the position of a non-involved observer, thinking about the picture in a neutral way". This could be achieved, for example, by reducing the personal involvement with 
This is a preprint of a revised manuscript submitted to PlosOne

208 the depicted situation, for instance by assuming a personal or physical distance; again, participants

209 were instructed to refrain from interpreting the situation as not real, attaching a different meaning to

210 the situation, or distracting themselves. The "distance" and "permit" instructions were chosen based

211 on previous work, which demonstrated their efficacy [10, 29]. All participants received written

212 instructions including examples, completed a training session outside the MR scanner, which took

213 about 10-15 min and consisted of 16 trials, and were subsequently interviewed about how they

214 implemented the proposed emotion regulation tactic.

Each of the four runs of the emotion regulation task consisted of 16 trials, encompassing four

216 trials for each condition. At the beginning of each trial, a picture was presented for $10 \mathrm{~s}$ (stimulation

217 phase, timepoint 1). During the initial $2 \mathrm{~s}$ of this period, a semi-transparent overlay was presented

218 across the center of the picture, which contained, as a single word, the instruction for either the

219 "permit" or the "distance" condition. Following the offset of the picture, a fixation cross was

220 presented for a variable period of 16-24 s (post-stimulation phase, timepoint 2). This rather long

221 period was inserted into the trial to provide the participants with a relaxation phase, and to allow the

222 return of the BOLD response to baseline levels. Altogether, the total duration of a single trial was, on

223 average, $30 \mathrm{~s}$. At the end of each run, participants were asked to give a rating of their retrospective

224 subjective arousal. For each experimental condition, participants rated on a continuous scale, how

225 much aroused they felt during the presentation of the negative and neutral images and the "distance"

226 and "permit" instructions, respectively. The scale for the continuous arousal scale ranged from -200

227 to 200 for technical reasons (screen coordinates). However, the particular choice of scale units and

228 limits did not affect the participants' ratings, since only verbal anchors were provided at either end of

229 the scale and participants had to move a slider to a position between those anchors that resembled

230 their arousal ("not at all aroused" vs. "very much aroused”). 
This is a preprint of a revised manuscript submitted to PlosOne

\section{Re-exposure task (timepoints 3 and 4)}

The re-exposure tasks at the end of session 1 (timepoint 3, 10 minutes later) and during session

2 (timepoint 4, 1 week later) consisted of the presentation of exactly those negative and neutral

234 pictures that participants had seen during the emotion regulation task. Since we wanted to investigate emotional reactivity, the presentation duration was shortened to $1000 \mathrm{~ms}$, in accordance with the emotional reactivity paradigm in the study by Walter and colleagues [9]. In contrast to the emotion regulation task, each presentation was followed by a variable inter-trial interval of $2 \mathrm{~s}$ to $12 \mathrm{~s}$, and participants should passively view the pictures. Specifically, they were instructed not to voluntarily change their emotional experience as they had done during the main experiment.

\section{Stimuli}

Stimuli were selected from the International Affective Picture System [IAPS, 30] and the

242 EmoPicS picture set [31]. We used two sets of negative pictures and two sets of neutral pictures (16

243 pictures per set) matched for content, arousal, and valence (mean valence negative pictures: set $1=$ 2.71 , set $2=2.65$; mean arousal negative pictures: set $1=5.85$, set $2=5.69$; mean valence neutral pictures: set $1=5.17$, set $2=5.13$; mean arousal neutral pictures: set $1=2.94$, set $2=2.96$ ). The negative pictures consisted primarily of depictions of animals, bodies, disaster, disgust, injuries, suffering, or violence, while the neutral pictures depicted various scenes, objects and people. The negative and neutral picture sets were matched with regard to depictions of faces, other parts of the body, single or multiple persons, animals, and inanimate objects. The assignment of the pictures to

250 either the "distance" or "permit" conditions was counterbalanced. In order to rule out any further 251 stimulus- or content-related confounds, two sets of negative and neutral pictures were used for one

252 half of the participants, and the other two negative and neutral sets were used for the other half of the 253 participants. Each set of pictures was arranged in two different sequences assigned to participants in 
This is a preprint of a revised manuscript submitted to PlosOne

254 an alternating fashion. For the emotion regulation task (timepoints 1 and 2), the order of stimuli was

255 pseudo-randomized within each sequence with the constraint that all experimental conditions

256 appeared equally within each experimental run and that no more than three presentations of the same 257 experimental condition occurred in succession. For the re-exposure tasks (timepoints 3 and 4), the 258 order of stimulus presentation was randomly shuffled. All pictures were projected to a screen located 259 at the rear end of the scanner and were viewed through a mirror attached to the head coil.

\section{Data acquisition}

Magnetic resonance (MR) imaging was done on a 3 Tesla scanner (Siemens Trio; Siemens

262 Erlangen, Germany), using a 12-channel head coil. Functional (T2*) MR images were acquired using an EPI sequence with 42 axial slices (slice thickness $2 \mathrm{~mm}$ ) per volume (TR $2410 \mathrm{~ms}$; TE $25 \mathrm{~ms}$; flip angle $80^{\circ}$; slice gap $1 \mathrm{~mm}$; field of view $192 \times 192 \mathrm{~mm}$; matrix size $\left.64 \times 64\right)$. In addition, anatomical (T1) images were acquired using an MPRAGE sequence consisting of 176 sagittal slices of $1 \mathrm{~mm}$ thickness (TR $1900 \mathrm{~ms}$; TE $2.26 \mathrm{~ms}$; flip angle 9; FOV $256 \mathrm{~mm} \times 256 \mathrm{~mm}$; matrix size $256 \times 256$ ).

\section{Data analysis}

\section{Behavioral data analyses}

Analysis of the subjective ratings and possible relations between subjective and physiological measures was performed with R 3.0.2 (http://r-project.org) including the ggplot2 package [32], and consisted of a two-way repeated-measures ANOVA with the factors picture (negative, neutral) and

272 regulation (permit, distance) with subsequent post-hoc $t$-tests for dependent samples. Due to technical 273 issues and incomplete measurements, ratings were not available from ten participants. 
This is a preprint of a revised manuscript submitted to PlosOne

\section{4 fMRI analyses}

Imaging data analysis for all tasks was performed using Matlab 7.4 (MathWorks, Natick, MA)

276

277

278 and SPM 8 (http://www.fil.ion.ucl.ac.uk/spm/software/spm8). After discarding the first 4 volumes of each run, preprocessing consisted of motion correction, coregistration of individual functional and anatomical data, spatial normalization of the anatomical images to the MNI template, application of the estimated transformation parameters to the coregistered functional images using a resampling resolution of $2 \times 2 \times 2 \mathrm{~mm}^{3}$, and spatial smoothing of the functional images (FWHM $8 \mathrm{~mm}$ ).

First-level statistical analysis of the emotion regulation task was performed using a general linear model with the experimental conditions (detailed below) as regressors and six additional motion regressors of no interest. To account for the notion that "rest" is not necessarily a true rest, especially not in emotionally challenging paradigms [22], we included not only the stimulation but also the post-stimulation phase in our model, starting with the offset of each picture and lasting for the same duration as the stimulation phase. This resulted in a first-level model with eight regressors of interest: we modeled the "permit neutral", "permit negative", "distance neutral", and "distance negative" conditions both for the stimulation (onset of picture presentation, timepoint 1) and poststimulation (offset of picture presentation, timepoint 2) phase. As the temporal dynamics of amygdala activation may differ from those in cortical regions, we conducted an additional sensitivity analysis for the amygdala, where activation was modeled by a stick function (transient response) in addition to a boxcar function (sustained response). This resulted in two different first-level models for the amygdala, whereas a single first-level model was used for all other brain regions. All regressors of interest were convolved with the canonical hemodynamic response function, and the default highpass filter for SPM8 (128s) was used. The imaging runs of the emotion regulation task were combined within one fixed-effects model. Parameter estimates for the contrasts of interest were averaged across runs, submitted to a second-level, random-effects analysis and evaluated using one-sample $t$-tests. 
This is a preprint of a revised manuscript submitted to PlosOne

The emotion regulation task follows a $2 * 2 * 2$ factorial design with the factors "picture"

299 (negative vs. neutral), "regulation" (permit vs. distance), and "time" (stimulation phase vs. post-

300 stimulation phase). For the stimulation phase, we analyzed the main effects of "picture" and

301 "regulation" as well as the interaction of "picture" and "regulation", which were the contrasts of

302 primary interest. Next, we repeated this analysis for the post-stimulation phase. Finally, we included

303 "time" as an additional factor into the model and computed the three-way interaction contrast for

304 "picture", "task", and "time" in order to characterize the changes in emotional regulation from

305 stimulation to post-stimulation. Additionally, the comparisons were restricted to either neutral or 306 negative pictures.

The first-level model of the re-exposure tasks (timepoints 3 and 4) included four regressors

308 for "negative permit", neutral permit", "negative distance", and "neutral distance" (assignment to

309 these conditions was based on the preceding emotion regulation task). The duration of all events was

310 set to $1000 \mathrm{~ms}$ as this was the duration of picture presentation. For statistical analysis, contrasts were

311 computed for the main and interaction effects of the "picture" and "preceding regulation" conditions,

312 and evaluated in a second-level analysis using one-sample t-tests.

Based on our a priori hypotheses, we employed two regions of interest (ROI), the left and

314 right amygdala as defined by the Harvard-Oxford Subcortical Structural Atlas within the FSL software package (https://fsl.fmrib.ox.ac.uk/fsl/fslwiki/Atlases). Masks were created from the

316 probabilistic segmentations at a threshold of 50\%. For statistical analyses within the amygdala, we

317 applied a threshold of $p=.05$ FWE after correction for small volume. For all other analyses, a voxel-

318 wise threshold of $p=.05$ FWE across the whole brain was applied. Activations were labeled using

319 the Harvard-Oxford Structural Atlases as well as the Anatomy Toolbox for SPM8 [33]. 
This is a preprint of a revised manuscript submitted to PlosOne

322 for all tasks. For this purpose, we extracted parameter estimates for all experimental conditions from

323 the individual first-level analyses using SPM8's spm_summarise() function and further analyzed these

324 data using repeated-measures ANOVAs with the factors picture, strategy, and/or time. In addition, for

325 the emotion regulation task, we extracted the activation time courses from these regions by using the 326 rfxplot toolbox (Gläscher 2009) to provide an illustration of the results of the model-based analyses.

\section{Results}

\section{Behavioral analysis of the stimulation phase (concurrent} 329 effects, timepoint 1)

The retrospective subjective arousal ratings after each run of the emotion regulation task demonstrated an interaction between picture and regulation $\left(F(1,37)=16.38, p<.001, \eta^{2}=.016\right)$ as well as main effects of picture $\left(F(1,37)=118.12, p<.001, \eta^{2}=.456\right)$ and regulation $(F(1,37)=$ $\left.107.15, p<.001, \eta^{2}=.160\right)$. On average, negative pictures were associated with higher subjective arousal ratings than neutral pictures. Pictures during "permit" were rated as more arousing than pictures during "distance" and this effect was more pronounced for negative than for neutral pictures. On a scale ranging from -200 to 200 , the experimental conditions had the following Mean \pm SD values: permit negative: $50.1 \pm 54.4$; distance negative: $-17.7 \pm 58.1$; permit neutral: $-75.4 \pm 71.0$; distance neutral: $-112.6 \pm 59.2$.

Neuronal activation differences during the stimulation phase

\section{(concurrent effects, timepoint 1)}

For both, the emotion regulation and the re-exposure tasks, we report the comparison of

342 pictures ("negative $>$ neutral" and vice versa) and regulation tactic ("distance > permit" and vice 
This is a preprint of a revised manuscript submitted to PlosOne

versa) as well as the interaction effects between picture and regulation. During the emotion regulation task, we consider these effects for both the stimulation phase (timepoint 1) and the post-stimulation phase (timepoint 2), separately. For simplicity, we only report interaction effects between regulation and time (stimulation to post-stimulation phase) in a joint analysis of the stimulation and poststimulation phase. Regarding the two analytic strategies for amygdala activation (transient and sustained responses), we point out when the choice of the analytical model matters; if not indicated otherwise, results hold for both strategies. Finally, we report effects of picture and regulation during re-exposure after $10 \mathrm{~min}$ (timepoint 3 ) and after 1 week (timepoint 4), separately.

\section{Activation differences between negative and neutral pictures}

Results of the analysis of activation differences during the stimulation phase are depicted in Fig 1. During the regulation phase, negative pictures elicited greater activation than neutral pictures in a large temporo-occipital cluster as well as a few additional brain regions (Table 1). Specifically, activation peaks were observed in the right fusiform gyrus, the left and right inferior temporal, right inferior frontal, left insula, posterior cingulate cortex as well as in the left and right amygdala. Greater activation during neutral than negative pictures was also observed, primarily in left and right superior temporal as well as left precentral regions.

- $\quad$ Insert Fig 1 here -

Fig 1. Whole-brain analysis of concurrent main effects of regulation (stimulation phase, timepoint 1). A. Permit $>$ Distance during the stimulation phase. B. Distance $>$ Permit during the stimulation phase.

Table 1. Activation maxima in the emotion regulation task during the stimulation phase (concurrent effects, timepoint 1). 
This is a preprint of a revised manuscript submitted to PlosOne

\begin{tabular}{|c|c|c|c|c|c|c|c|}
\hline \multicolumn{8}{|c|}{ Neutral Stim Phase $>$ Negative Stim Phase } \\
\hline 248 & 0.001 & 6.52 & $<0.001$ & -32 & -30 & 62 & Left Precentral Gyrus \\
\hline 129 & 0.002 & 6.23 & $<0.001$ & -58 & -16 & 2 & Left Superior Temporal Gyrus \\
\hline 38 & 0.004 & 6.08 & $<0.001$ & 58 & -4 & -4 & Right Superior Temporal Gyrus \\
\hline 32 & 0.005 & 5.99 & $<0.001$ & 12 & -20 & 48 & Right SMA \\
\hline 47 & 0.005 & 5.95 & $<0.001$ & 44 & -64 & 50 & Right Angular Gyrus \\
\hline 39 & 0.005 & 5.94 & $<0.001$ & 42 & -14 & 20 & Right Rolandic Operculum \\
\hline 42 & 0.011 & 5.69 & $<0.001$ & -34 & -20 & 48 & Left Precentral Gyrus \\
\hline
\end{tabular}

\begin{tabular}{|c|c|c|c|c|c|c|c|}
\hline \multicolumn{8}{|c|}{ Negative Stim Phase > Neutral Stim Phase } \\
\hline 6265 & $<0.001$ & 13.63 & $<0.001$ & 42 & -46 & -14 & Right Fusiform Gyrus \\
\hline 5965 & $<0.001$ & 12.28 & $<0.001$ & -40 & -46 & -16 & Left Inferior Temporal Gyrus \\
\hline 1876 & $<0.001$ & 10.23 & $<0.001$ & 44 & 18 & 24 & Right Inferior Frontal Gyrus \\
\hline 1963 & $<0.001$ & 10.15 & $<0.001$ & 14 & -20 & -10 & N/A \\
\hline 339 & $<0.001$ & 7.96 & $<0.001$ & -32 & 18 & -18 & Left Insula Lobe \\
\hline 134 & 0.001 & 6.62 & $<0.001$ & -2 & -50 & 26 & Left Posterior Cingulate Cortex \\
\hline 163 & 0.001 & 6.61 & $<0.001$ & 2 & -56 & -36 & Cerebellar Vermis \\
\hline 25 & 0.006 & 5.91 & $<0.001$ & 6 & 48 & -20 & Right Gyrus Rectus \\
\hline 36 & 0.016 & 5.56 & $<0.001$ & 0 & -54 & 44 & Left Precuneus \\
\hline 164 & $<0.001$ & 6.91 & $<0.001$ & -24 & -8 & -14 & Left Amygdala (ROI) \\
\hline 183 & $<0.001$ & 7.70 & $<0.001$ & 20 & -6 & -12 & Right Amygdala (ROI) \\
\hline 190 & $<0.001$ & 7.63 & $<0.001$ & -20 & -8 & -12 & Left Amygdala (ROI, stick model) \\
\hline 211 & $<0.001$ & 7.86 & $<0.001$ & 18 & -6 & -12 & Right Amygdala (ROI, stick model) \\
\hline
\end{tabular}

\begin{tabular}{cccccccc}
\hline \multicolumn{3}{l}{ Main effect regulation } \\
\multicolumn{7}{l}{ Distance Stim Phase $>$ Permit Stim Phase } \\
\hline 2957 & $<0.001$ & 9.73 & $<0.001$ & 36 & 28 & 40 & Right Middle Frontal Gyrus \\
1967 & $<0.001$ & 9.65 & $<0.001$ & 54 & -52 & 38 & Right Inferior Parietal Lobule \\
274 & $<0.001$ & 7.35 & $<0.001$ & -40 & 22 & 34 & Left Middle Frontal Gyrus \\
302 & $<0.001$ & 7.07 & $<0.001$ & 12 & -72 & 38 & Right Cuneus \\
858 & $<0.001$ & 6.88 & $<0.001$ & -42 & -50 & 44 & Left Inferior Parietal Lobule
\end{tabular}


This is a preprint of a revised manuscript submitted to PlosOne

$\begin{array}{cccccccl}214 & <0.001 & 6.81 & <0.001 & -12 & -76 & 46 & \text { Left Precuneus } \\ 111 & 0.001 & 6.42 & <0.001 & 6 & -38 & 24 & \text { Right Posterior Cingulate Cortex } \\ 119 & 0.001 & 6.41 & <0.001 & 66 & -20 & -10 & \text { Right Middle Temporal Gyrus } \\ 173 & 0.002 & 6.26 & <0.001 & 36 & 20 & 0 & \text { Right Insula Lobe } \\ 129 & 0.003 & 6.23 & <0.001 & -32 & 46 & 8 & \text { Left Middle Frontal Gyrus } \\ 71 & 0.003 & 6.17 & <0.001 & 10 & -34 & 42 & \text { Right Middle Cingulate Cortex } \\ 70 & 0.009 & 5.80 & <0.001 & 44 & 48 & -4 & \text { Right Inferior Frontal Gyrus } \\ 67 & 0.011 & 5.74 & <0.001 & -58 & 24 & 6 & \text { Left Inferior Frontal Gyrus }\end{array}$

Permit Stim Phase > Distance Stim Phase

\begin{tabular}{rrrrrrrl}
\hline 159 & 0.001 & 6.43 & $<0.001$ & -22 & -96 & -8 & Left Inferior Occipital Gyrus \\
122 & 0.002 & 6.25 & $<0.001$ & 28 & -94 & 0 & Right Middle Occipital Gyrus \\
27 & 0.016 & 3.40 & 0.001 & -18 & -10 & -12 & Left Amygdala (ROI) \\
28 & 0.015 & 3.50 & 0.001 & 18 & -8 & -12 & Right Amygdala (ROI) \\
122 & 0.001 & 4.33 & $<0.001$ & -18 & -6 & -12 & Left Amygdala (ROI, stick model) \\
115 & 0.002 & 4.22 & $<0.001$ & 22 & -10 & -14 & Right Amygdala (ROI, stick model) \\
\hline
\end{tabular}

Interaction effect picture $\mathrm{X}$ regulation

(DistanceNeutral Stim Phase > PermitNeutral Stim Phase) > (DistanceNegative Stim Phase > PermitNegative Stim Phase)

\begin{tabular}{llllllll}
\hline 13 & 0.044 & 3.05 & 0.002 & -18 & -10 & -12 & Left Amygdala (ROI, stick model) \\
10 & 0.031 & 3.29 & 0.001 & 32 & -2 & -22 & Right Amygdala (ROI, stick model) \\
\hline
\end{tabular}

(PermitNeutral Stim Phase $>$ DistanceNeutral Stim Phase) $>$ (PermitNegative Stim Phase $>$ DistanceNegative Stim Phase)

$\begin{array}{llllllll}5 & 0.023 & 3.33 & 0.001 & 22 & -4 & -26 & \text { Right Amygdala (ROI) }\end{array}$

\section{Regulation effect negative pictures only}

\begin{tabular}{|c|c|c|c|c|c|c|c|}
\hline \multicolumn{8}{|c|}{ DistanceNegative Stim Phase $>$ PermitNegative Stim Phase } \\
\hline 1590 & $<0.001$ & 8.76 & $<0.001$ & 52 & -48 & 36 & Right Angular Gyrus \\
\hline 2116 & $<0.001$ & 8.71 & $<0.001$ & 42 & 26 & 38 & Right Middle Frontal Gyrus \\
\hline 478 & $<0.001$ & 6.89 & $<0.001$ & -52 & 38 & -8 & Left Inferior Frontal Gyrus \\
\hline 147 & 0.001 & 6.62 & $<0.001$ & -42 & 22 & 34 & Left Middle Frontal Gyrus \\
\hline 102 & 0.001 & 6.43 & $<0.001$ & 62 & -36 & -20 & Right Inferior Temporal Gyrus \\
\hline 294 & 0.003 & 6.21 & $<0.001$ & -48 & -50 & 44 & Left Inferior Parietal Lobule \\
\hline 113 & 0.004 & 6.11 & $<0.001$ & 42 & 18 & -10 & Right Insula Lobe \\
\hline
\end{tabular}


This is a preprint of a revised manuscript submitted to PlosOne

$\begin{array}{ccccccc}149 & 0.004 & 6.06 & <0.001 & -30 & 44 & 8 \text { Left Middle Frontal Gyrus } \\ 74 & 0.005 & 6.04 & <0.001 & 12 & -66 & 40 \text { Right Precuneus }\end{array}$

PermitNegative Stim Phase $>$ DistanceNegative Stim Phase

\begin{tabular}{rrrrrrr}
\hline 5 & 0.041 & 3.06 & 0.002 & 18 & -8 & -12 Right Amygdala (ROI) \\
148 & 0.001 & 4.68 & $<0.001$ & -20 & -8 & -14 Left Amygdala (ROI, stick model) \\
100 & 0.006 & 3.94 & $<0.001$ & 22 & -10 & -14 Right Amygdala (ROI, stick model)
\end{tabular}

Regulation effect neutral pictures only

PermitNeutral Stim Phase > DistanceNeutral Stim Phase

\begin{tabular}{|c|c|c|c|c|c|c|}
\hline 205 & 0.001 & 6.45 & $<0.001$ & 36 & 40 & 32 Right Middle Frontal Gyrus \\
\hline 192 & 0.002 & 6.36 & $<0.001$ & 60 & -42 & 30 Right Supramarginal Gyrus \\
\hline \multicolumn{7}{|c|}{ PermitNeutral Stim Phase $>$ DistanceNeutral Stim Phase } \\
\hline 267 & 0.001 & 6.69 & $<0.001$ & -20 & -96 & -6 Left Inferior Occipital Gyrus \\
\hline 182 & 0.003 & 6.21 & $<0.001$ & 26 & -88 & 6 Right Middle Occipital Gyrus \\
\hline 12 & 0.038 & 3.02 & 0.002 & -18 & -8 & -18 Left Amygdala (ROI) \\
\hline 20 & 0.045 & 3.02 & 0.002 & 20 & -8 & -16 Right Amygdala (ROI) \\
\hline 12 & 0.014 & 3.50 & 0.001 & -16 & -4 & -14 Left Amygdala (ROI, stick model) \\
\hline 50 & 0.02 & 3.42 & 0.001 & 18 & -8 & -18 Right Amygdala (ROI, stick model) \\
\hline
\end{tabular}

Abbreviations: $\mathrm{k}=$ spatial extent, $p_{\mathrm{FWE}}=p$-values corrected for multiple comparisons $(\mathrm{FWE}), p_{\text {unc. }}=$ uncorrected $p$-values, $t=t$ statistics, $\mathrm{x}, \mathrm{y}, \mathrm{z}=$ MNI coordinates. ROI indicates that an activation peak was observed within the left or right amygdala region of interest.

\section{Activation differences between distancing and permitting emotions}

Besides the main effect of picture, there was also a main effect of regulation (Table 1): Clusters

of greater activation during distance as compared to permit were observed in the left and right inferior and middle frontal gyrus, left and right inferior parietal lobe and the precuneus, among others.

367 Reverse effects, i.e. greater activation during permit than during distance, were exclusively observed

368 in the left and right amygdala and the occipital cortex. When comparing responses to negative pictures

369 (Table 1), greater activation for distance vs. permit was primarily observed in the right frontal and 370 parietal cortex. Conversely, greater activation for permit vs. distance was present in the left and right 
This is a preprint of a revised manuscript submitted to PlosOne

371 amygdala and occipital cortex. Interestingly, similar activation patterns also emerged when only 372 neutral pictures were considered (Table 1).

\section{Interaction effects between picture and regulation}

Finally, interaction effects of picture and regulation were observed: the distance vs. permit difference was greater for negative than for neutral pictures in the left and right amygdala for transient responses, while contradictory results were observed for sustained responses (Table 1).

In order to further characterize the effects observed during the voxel-based analysis, we conducted an additional analysis of summary statistics based on the left and right amygdala ROIs (see

Fig 2Fehler! Verweisquelle konnte nicht gefunden werden.). We observed greater activation for negative than neutral pictures as well as a decrease of this activation during distance vs. permit. That is, there were significant picture and regulation main effects during the stimulation phase, but no interaction effects (left amygdala: picture: $F(1,45)=30.56, p<.001, \eta 2=.112$; strategy: $F(1,45)=$ picture: $F(1,45)=26.65, p<.001, \eta^{2}=.109$; strategy: $F(1,45)=9.02, p=.004, \eta^{2}=.037$; picture $\times$ strategy $\left.F(1,45)=1.62, p=.210, \eta^{2}=.003\right)$. 


\section{Neuronal activation differences during the post-stimulation}

Picture and regulation effects were also present during the post-stimulation phase (Table 2):

392 For both, the permit and distance condition, there was a greater activation for negative than neutral

393 pictures in the amygdala ROI, but not in any other brain region. Across picture conditions, greater

394 activation was observed after distance than after permit in brain regions such as the occipital cortex,

395 the precuneus, and also the amygdala (for sustained responses). Similar effects emerged when the

396 comparison was restricted to negative pictures and even neutral picture (primarily for sustained

397 responses). Conversely, we did not observe any activation difference for the reverse contrast, that is

398 there was no greater activation after permit vs. after distance in the post-stimulation phase. No

399 interaction effects between picture and strategy were present during the post-stimulation phase.

Table 2. Activation maxima in the emotion regulation task during the post-stimulation phase (immediate effects, timepoint 2).

\begin{tabular}{|c|c|c|c|c|c|c|}
\hline $\mathbf{k}$ & $p_{\text {FWE }}$ & $t$ & $p_{\text {unc. }}$ & $\mathbf{x}$ & $\mathbf{y}$ & z label \\
\hline \multicolumn{7}{|c|}{ Main effect picture } \\
\hline \multicolumn{7}{|c|}{ Neutral Post-Stim Phase > Negative Post-Stim Phase } \\
\hline \multicolumn{7}{|l|}{ No results } \\
\hline \multicolumn{7}{|c|}{ Negative Post-Stim Phase > Neutral Post-Stim Phase } \\
\hline 49 & 0.028 & 3.16 & 0.001 & -22 & -12 & -12 Left Amygdala (ROI) \\
\hline 27 & 0.033 & 3.16 & 0.001 & 28 & -6 & -14 Right Amygdala (ROI \\
\hline 75 & 0.004 & 3.94 & $<0.001$ & -26 & -6 & -18 Left Amygdala (ROI, stick model) \\
\hline 105 & 0.007 & 3.82 & $<0.001$ & 24 & -6 & -14 Right Amygdala (ROI, stick model) \\
\hline
\end{tabular}

Main effect regulation

Distance Post-Stim Phase > Permit Post-Stim Phase

$\begin{array}{lllllll}75 & 0.004 & 6.03 & <0.001 & -42 & -68 & 4\end{array}$ Left Middle Occipital Gyrus


This is a preprint of a revised manuscript submitted to PlosOne

$\begin{array}{llllllll}46 & 0.006 & 5.87 & <0.001 & -44 & -52 & -24 & \text { Left Inferior Temporal Gyrus } \\ 37 & 0.014 & 5.59 & <0.001 & -4 & -76 & 48 \text { Left Precuneus } \\ 53 & 0.024 & 5.39 & <0.001 & -42 & -54 & 54 \text { Left Inferior Parietal Lobule } \\ 18 & 0.028 & 3.12 & 0.002 & -26 & -12 & -14 \quad \text { Left Amygdala (ROI) } \\ 93 & 0.002 & 4.21 & <0.001 & 32 & -2 & -20 \text { Right Amygdala (ROI) }\end{array}$

Permit Post-Stim Phase > Distance Post-Stim Phase

No results

Interaction effect picture $X$ regulation

(DistanceNeutral Post-Stim Phase $>$ PermitNeutral Post-Stim Phase) $>$ (DistanceNegative Post-Stim Phase $>$ PermitNegative PostStim Phase)

No results

(PermitNeutral Post-Stim Phase $>$ DistanceNeutral Post-Stim Phase) $>$ (PermitNegative Post-Stim Phase $>$ DistanceNegative PostStim Phase)

No results

\section{Regulation effect negative pictures only}

DistanceNegative Post-Stim Phase > PermitNegative Post-Stim Phase

\begin{tabular}{rlllllll}
\hline 6 & 0.026 & 3.15 & 0.001 & -26 & -10 & -16 Left Amygdala (ROI) \\
41 & 0.015 & 3.44 & 0.001 & 24 & -8 & -18 Right Amygdala (ROI) \\
6 & 0.059 & 2.94 & 0.003 & 24 & -8 & -18 Right Amygdala (ROI, stick model) \\
\hline
\end{tabular}

PermitNegative Post-Stim Phase $>$ DistanceNegative Post-Stim Phase

No results

\section{Regulation effect neutral pictures only}

DistanceNeutral Post-Stim Phase > PermitNeutral Post-Stim Phase

\begin{tabular}{|c|c|c|c|c|c|c|}
\hline 7 & 0.012 & 3.54 & $<0.001$ & -32 & 0 & -18 Left Amygdala (ROI) \\
\hline 49 & 0.033 & 3.16 & 0.001 & 32 & -2 & -22 Right Amygdala (ROI) \\
\hline
\end{tabular}

PermitNeutral Post-Stim Phase > DistanceNeutral Post-Stim Phase

No results

Abbreviations: $\mathrm{k}=$ spatial extent, $p_{\mathrm{FWE}}=p$-values corrected for multiple comparisons $(\mathrm{FWE}), p_{\text {unc. }}=$ uncorrected $p$-values, $t=t$ statistics, $\mathrm{x}, \mathrm{y}, \mathrm{z}=\mathrm{MNI}$ coordinates. ROI indicates that an activation peak was observed within the left or right amygdala region of interest. 


\section{Neuronal interaction effects of regulation (permit, distance) and}

404

\section{time (stimulation, post-stimulation)}

When time was included as additional factor in the analysis (Table 3), that is, when considering changes from stimulation to post-stimulation phase, interaction effects between regulation and time were observed in several regions. In the right occipital cortex as well as the left and right amygdala, the effect permit vs. distance was greater during stimulation than during poststimulation. Conversely, in the right superior frontal gyrus and the right inferior parietal lobe, the reverse effect, that is distance vs. permit, was greater during stimulation than during post-stimulation. Similar patterns emerged when the analysis was restricted to negative pictures and even neutral pictures (for the latter, only in sustained responses).

This analysis was complemented by an additional analysis of summary statistics based on the left and right amygdala ROIs in order to further characterize the voxel-based effects. Specifically, we conducted an analysis for negative pictures that also took the effect of time into account. We found significant regulation-by-time interactions in the left and right amygdala (Fig 3), indicating greater activation in the permit condition than the distance condition during the stimulation phase, but not during the post-stimulation phase (left amygdala: strategy: $F(1,45)=2.13, p=.151, \eta^{2}=.008$; Time: $F(1,45)=0.55, p=.461, \eta^{2}=.004 ;$ strategy $\times$ Time: $F(1,45)=11.86, p=.001, \eta^{2}=.029 ;$ right amygdala: strategy: $F(1,45)=1.33, p=.254, \eta^{2}=.005$; Time: $F(1,45)=0.94, p=.338, \eta^{2}=.008$; strategy $\times$ Time: $\left.F(1,45)=11.51, p=.001, \eta^{2}=.025\right)$.

Table 3. Immediate aftereffects of emotion regulation (interaction effects of regulation (permit, distance) and time (stimulation/timepoint 1, post-stimulation/timepoint 2).
$\mathbf{k}$
$p_{\text {FWE }}$
punc.
$\mathbf{x}$
$\mathbf{y}$
z label

\section{Interaction effect regulation $\mathrm{X}$ time}


This is a preprint of a revised manuscript submitted to PlosOne

\begin{tabular}{|c|c|c|c|c|c|c|}
\hline \multicolumn{7}{|c|}{ (Distance Stim Phase $>$ Permit Stim Phase) $>$ (Distance Post-Stim Phase $>$ Permit Post-Stim Phase) } \\
\hline 95 & 0.001 & 6.48 & $<0.001$ & 60 & -50 & 46 Right Inferior Parietal Lobule \\
\hline 31 & 0.006 & 5.94 & $<0.001$ & 20 & 8 & 64 Right Superior Frontal Gyrus \\
\hline \multicolumn{7}{|c|}{ (Permit Stim Phase $>$ Distance Stim Phase) $>$ (Permit Post-Stim Phase $>$ Distance Post-Stim Phase) } \\
\hline 148 & 0.003 & 6.22 & $<0.001$ & -18 & -96 & -10 Left Inferior Occipital Gyrus \\
\hline 127 & 0.005 & 6.00 & $<0.001$ & 14 & -100 & 0 Right Calcarine Gyrus \\
\hline 31 & 0.007 & 5.90 & $<0.001$ & 0 & 2 & -10 Subcallosal Cortex \\
\hline 39 & 0.008 & 5.84 & $<0.001$ & 34 & -86 & -14 Right Inferior Occipital Gyrus \\
\hline 161 & 0.002 & 4.29 & $<0.001$ & -18 & -12 & -14 Left Amygdala (ROI) \\
\hline 226 & $<0.001$ & 4.97 & $<0.001$ & 32 & -2 & -20 Right Amygdala (ROI) \\
\hline 134 & $<0.001$ & 4.97 & $<0.001$ & -26 & -12 & -14 Left Amygdala (ROI, stick model) \\
\hline 144 & 0.001 & 4.64 & $<0.001$ & 24 & -8 & -18 Right Amygdala (ROI, stick model) \\
\hline
\end{tabular}

\section{Interaction effect regulation $\mathrm{X}$ time, negative pictures only}

(DistanceNegative Stim Phase > PermitNegative Stim Phase) > (DistanceNegative Post-Stim Phase > PermitNegative Post-Stim Phase)
68
0.001
6.6
$<0.001$
$18 \quad 12$
62 Right Superior Frontal Gyrus

(PermitNegative Stim Phase $>$ DistanceNegative Stim Phase) $>$ (PermitNegative Post-Stim Phase $>$ DistanceNegative Post-Stim Phase)

\begin{tabular}{rrrrrrrl}
\hline 77 & 0.004 & 3.98 & $<0.001$ & -24 & -12 & -14 Left Amygdala (ROI) \\
163 & 0.003 & 4.12 & $<0.001$ & 24 & -12 & -12 Right Amygdala (ROI) \\
114 & $<0.001$ & 5.40 & $<0.001$ & -28 & -10 & -16 Left Amygdala (ROI, stick model) \\
146 & $<0.001$ & 5.22 & $<0.001$ & 24 & -8 & -18 Right Amygdala (ROI, stick model) \\
\hline
\end{tabular}

\section{Interaction effect regulation $\mathrm{X}$ time, neutral pictures only}

(DistanceNeutral Stim Phase > PermitNeutral Stim Phase) $>$ (DistanceNeutral Post-Stim Phase > PermitNeutral Post-Stim Phase)

\section{No results}

(PermitNeutral Stim Phase $>$ DistanceNeutral Stim Phase) $>$ (PermitNeutral Post-Stim Phase $>$ DistanceNeutral Post-Stim Phase)

\begin{tabular}{|c|c|c|c|c|c|c|}
\hline 57 & 0.006 & 5.92 & $<0.001$ & -20 & -96 & -8 Left Inferior Occipital Gyrus \\
\hline 69 & 0.013 & 3.49 & 0.001 & -16 & -10 & -16 Left Amygdala (ROI, stick model) \\
\hline 187 & 0.001 & 4.41 & $<0.001$ & 32 & -2 & -20 Right Amygdala (ROI, stick model) \\
\hline
\end{tabular}


Abbreviations: $\mathrm{k}=$ spatial extent, $p_{\mathrm{FWE}}=p$-values corrected for multiple comparisons (FWE), $p_{\text {unc. }}=$ uncorrected $p$-values, $t=t$ statistics, $\mathrm{x}, \mathrm{y}, \mathrm{z}=\mathrm{MNI}$ coordinates. ROI indicates that an activation peak was observed within the left or right amygdala region of interest.

Fig 3. Summary statistics for the amygdala ROI during the stimulation phase (timepoint 1 ) and the post-stimulation phase (immediate aftereffects, timepoint 2). This analysis is limited to negative stimuli.

\section{Activation time-courses during the stimulation- and post-stimulation}

phase (timepoint 1, timepoint 2)

The temporal course of activation in the left and right amygdala was investigated in a post-

hoc analysis (Fig 4). It is noteworthy that in this descriptive analysis, an initial peak during the

regulation period was followed by a second peak during the post-stimulation period. While the first peak, which shows roughly similar activation levels for all four conditions.

Fig 4. Activation time-courses in the left and right amygdala during stimulation (timepoint 1) and post-stimulation phase (immediate aftereffects, timepoint 2 ). The shaded area indicates the stimulation phase

\section{Neuronal activation differences during the re-exposure tasks}

Re-exposure after 10 minutes (short-term effects, timepoint 3)

During re-exposure after 10 minutes, picture main effects were observed in the posterior cingulate gyrus, the fusiform gyrus, the right temporal gyrus, the left occipital gyrus, and the left

439 within the left and right amygdala when the analysis was restricted to negative pictures (Fig 5; Table 
This is a preprint of a revised manuscript submitted to PlosOne

\section{Re-exposure after 1 week (long-term effects, timepoint 4)}

Re-exposure after 1 week led to greater activation for negative than for neutral pictures in

443 several brain regions, including the right inferior temporal gyrus, left occipital, fusiform, and inferior

444 frontal gyrus, and the left and right amygdala (Table 5). Again, there was an effect of the previous

445 regulation condition: greater activation for previously distanced pictures was present in the right

446 amygdala (Fig 5). Restricting this comparison to negative pictures did not yield any significant effect,

447 but a small effect of the same kind appeared for neutral pictures (Table 5). No other effect of interest

448 in this analysis exceeded the whole-brain threshold.

Fig 5. Summary statistics for the amygdala ROI during re-exposure after 10 min (short-term effects, timepoint 3 ) and after 1 week (long-term effects, timepoint 4).

Table 4. Activation maxima during re-exposure after 10 minutes (short-term effects, timepoint 3).

\begin{tabular}{|c|c|c|c|c|c|c|c|}
\hline $\mathbf{k}$ & $p_{\text {FWE }}$ & $t$ & $p_{\text {unc. }}$ & $\mathbf{x}$ & $\mathbf{y}$ & $\mathbf{z}$ & label \\
\hline \multicolumn{8}{|c|}{ Main effect picture } \\
\hline \multicolumn{8}{|c|}{ Neutral $>$ Negative } \\
\hline \multicolumn{8}{|l|}{ No results } \\
\hline \multicolumn{8}{|c|}{ Negative $>$ Neutral } \\
\hline 148 & $<0.001$ & 8.21 & $<0.001$ & -8 & -18 & 30 & Left Posterior Cingulate Cortex \\
\hline 428 & $<0.001$ & 7.96 & $<0.001$ & 42 & -50 & -16 & Right Fusiform Gyrus \\
\hline 366 & $<0.001$ & 7.16 & $<0.001$ & -42 & -48 & -22 & Left Fusiform Gyrus \\
\hline 167 & 0.001 & 6.83 & $<0.001$ & 50 & -70 & 12 & Right Middle Temporal Gyrus \\
\hline 85 & 0.006 & 6.23 & $<0.001$ & -44 & -80 & -2 & Left Middle Occipital Gyrus \\
\hline 8 & 0.005 & 3.97 & $<0.001$ & -16 & -8 & -14 & Left Amygdala (ROI) \\
\hline \multicolumn{8}{|c|}{ Main effect regulation } \\
\hline Distance & & & & & & & \\
\hline
\end{tabular}


This is a preprint of a revised manuscript submitted to PlosOne

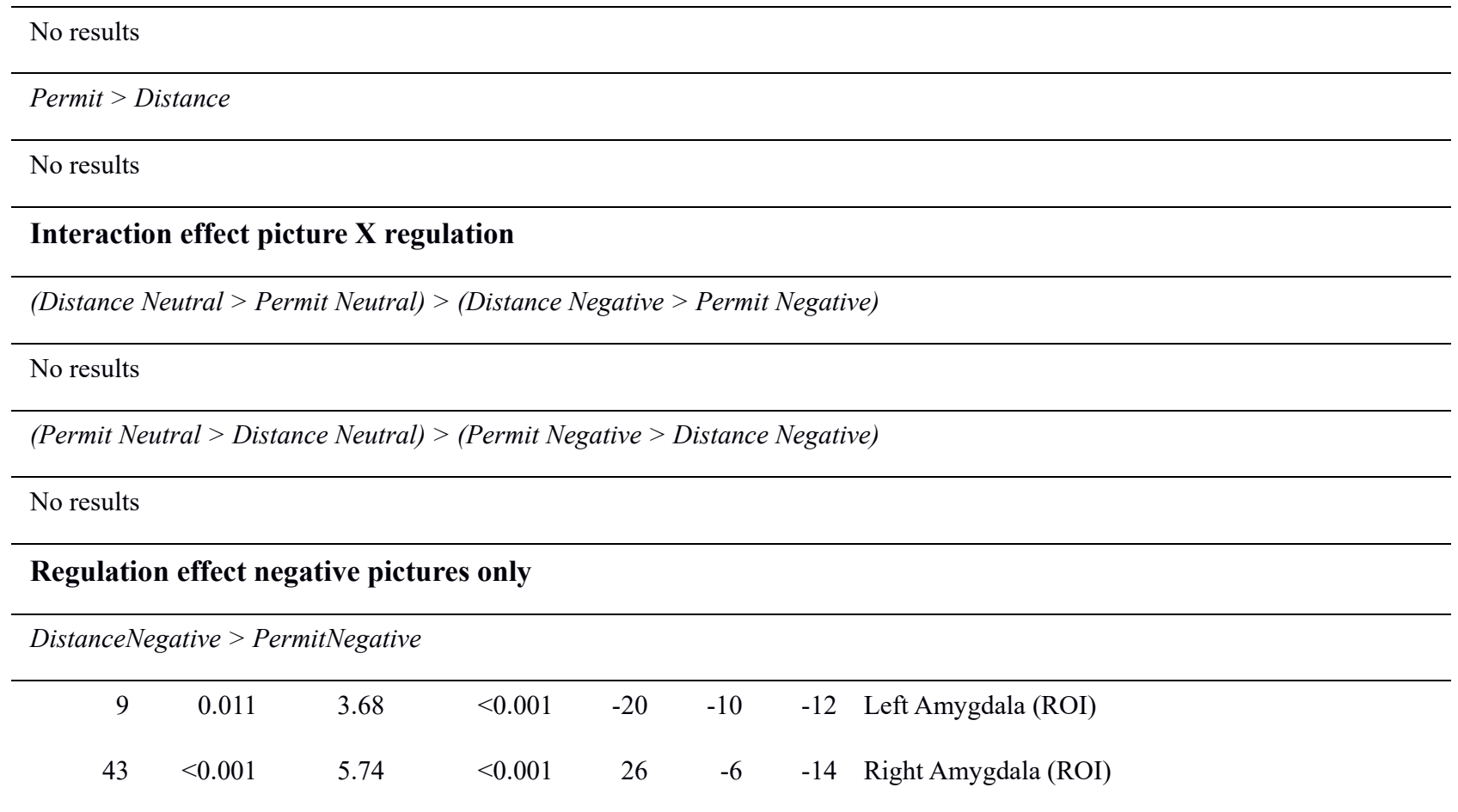

PermitNegative $>$ DistanceNegative

\title{
No results
}

\section{Regulation effect neutral pictures only}

DistanceNeutral $>$ PermitNeutral

\author{
No results \\ PermitNeutral $>$ DistanceNeutral
}

\section{No results}

Abbreviations: $\mathrm{k}=$ spatial extent, $p_{\mathrm{FWE}}=p$-values corrected for multiple comparisons $(\mathrm{FWE}), p_{\text {unc. }}=$ uncorrected $p$-values, $t=t$ statistics, $\mathrm{x}, \mathrm{y}, \mathrm{z}=\mathrm{MNI}$ coordinates. ROI indicates that an activation peak was observed within the left or right amygdala region of interest.

Table 5. Activation maxima during re-exposure after 1 week (long-term effects, timepoint 4).

\begin{tabular}{|c|c|c|c|c|}
\hline$p_{\text {FWE }}$ & $t$ & punc. & $\mathbf{x}$ & z label \\
\hline \multicolumn{5}{|l|}{ Main effect picture } \\
\hline \multicolumn{5}{|l|}{ Neutral $>$ Negative } \\
\hline \multicolumn{5}{|l|}{ No results } \\
\hline \multicolumn{5}{|l|}{ Negative $>$ Neutral } \\
\hline$<0.001$ & 8.97 & $<0.001$ & 44 & -14 Right Inferior Temporal Gyrus \\
\hline
\end{tabular}


This is a preprint of a revised manuscript submitted to PlosOne

$\begin{array}{rrrrrrrl}122 & <0.001 & 8.68 & <0.001 & -32 & -76 & 20 & \text { Left Middle Occipital Gyrus } \\ 85 & <0.001 & 8.01 & <0.001 & 18 & -6 & -14 & \text { Right Hippocampus } \\ 150 & 0.004 & 6.99 & <0.001 & -40 & -50 & -12 & \text { Left Fusiform Gyrus } \\ 84 & 0.005 & 6.90 & <0.001 & -30 & 22 & -20 & \text { Left Inferior Frontal Gyrus } \\ 79 & <0.001 & 5.23 & <0.001 & -24 & -4 & -14 & \text { Left Amygdala (ROI) } \\ 149 & <0.001 & 8.01 & <0.001 & 18 & -6 & -14 & \text { Right Amygdala (ROI) }\end{array}$

\section{Main effect regulation}

Distance $>$ Permit

\begin{tabular}{llllllll}
\hline 23 & 0.005 & 4.28 & $<0.001$ & 24 & 0 & -22 & Right Amygdala (ROI) \\
\hline
\end{tabular}

Permit $>$ Distance

No results

Interaction effect picture $\mathrm{X}$ regulation

(Distance Neutral $>$ Permit Neutral) $>$ (Distance Negative $>$ Permit Negative)

No results

(Permit Neutral $>$ Distance Neutral) $>$ (Permit Negative $>$ Distance Negative)

No results

\section{Regulation effect negative pictures only}

DistanceNegative $>$ PermitNegative

No results

PermitNegative $>$ DistanceNegative

No results

Regulation effect neutral pictures only

DistanceNeutral > PermitNeutral

\begin{tabular}{llllllll}
\hline 16 & 0.016 & 3.76 & $<0.001$ & 24 & -4 & -20 & Right Amygdala (ROI)
\end{tabular}

PermitNeutral $>$ DistanceNeutral

No results

Abbreviations: $\mathrm{k}=$ spatial extent, $p_{\mathrm{FWE}}=p$-values corrected for multiple comparisons $(\mathrm{FWE}), p_{\text {unc. }}=$ uncorrected $\mathrm{p}$-values, $t=t$ statistics, $\mathrm{x}, \mathrm{y}, \mathrm{z}=\mathrm{MNI}$ coordinates. ROI indicates that an activation peak was observed within the left or right amygdala region of interest. 
This is a preprint of a revised manuscript submitted to PlosOne

\section{Discussion}

This study presents a systematic investigation of emotional processing and regulation across

458 two picture types and regulation conditions and immediate as well as short- and long-term time scales.

459 Its main results can be summarized as follows: During stimulation (at timepoint 1) negative pictures

460 elicited a strong response in affective regions of the brain, most prominently the amygdala, but also

461 the insula and cingulate cortex. Volitional emotion regulation, as implemented by objective

462 distancing, led to a decrease of this response, and to an increase of activation in the right middle

463 frontal and inferior parietal cortex. During the stimulation phase, distancing-related activation in

464 cortical regions appeared as a sustained response, whereas the reverse effect, that is the down-

465 regulation of the amygdala, appeared mainly as a transient response, in particular for negative

466 pictures. Regarding paradoxical immediate aftereffects in the amygdala (at timepoint 2), statistically

467 significant interaction effects between time and regulation indicate that higher levels of amygdala

468 activation in the permit condition during the stimulation phase reversed to higher activation in the

469 distance condition during the post-stimulation phase. Further (paradoxical) task-rest interactions for

470 negative pictures were observed in the occipital cortex and the ventromedial frontal / subgenual

471 cingulate cortex. Previous emotion regulation had an impact on amygdala activation both at timepoint

4723 (after 10 minutes) and timepoint 4 (after one week): Activation within the amygdala was higher if

473 the participants had previously (in the emotion regulation task) been instructed to distance from the

474 picture as compared to permit all upcoming emotion. 
 \\ Effects of emotional processing and regulation at timepoint 1}

476

\section{Concurrent emotion regulation effects - Replication and extension}

A key result of the present study is the replication of canonical emotion regulation effects, in particular the down-regulation of the amygdala and the concurrent activation of a right frontoparietal control network during distancing from negative pictures. This confirms several previous reports [10, $20,34]$. This pattern of replications hints at the robustness of our experimental paradigm and can serve as a basis for a systematic extension, as will be discussed below, for the exploration of activation changes in other cortical and subcortical regions, and for the incorporation of additional experimental variables such as time.

A second result of this study is that the observed effects extend beyond the regions that are commonly implicated in emotion regulation. Specifically, negative pictures were not only associated with amygdala and insula activation, but also with activation in the inferior temporal gyrus and the occipital cortex, the latter was also observed during the permit condition. During the distance negative condition, left- (instead of right-) hemispheric activation in the inferior and middle frontal gyrus specifically was an unexpected observation with respect to our hypotheses and previous results [10].

Other authors have noted that emotion regulation - as any complex psychological function does not rely on single brain regions [for network analyses see for instance 17, 35, 36]. Emotion regulation is a heterogeneous construct that overlaps to a certain extent with other cognitive domains: for example, Messina, Bianco, Sambin and Viviani (37) distinguish between executive and semantic processes during reappraisal; they suggest that executive functions serve a general role, while semantic regions in the temporal and parietal cortex should be exclusively activated during reappraisal via perspective-taking. With regard to our finding of additional left-hemispheric frontal cortical activation, it is possible that inner speech is a component of distancing via self-instruction 
This is a preprint of a revised manuscript submitted to PlosOne

498 that results in selective recruitment of left frontal regions [38] in addition to a more general, right-

499 hemispheric control network. Further support for the idea that higher-level regulation strategies

500 recruit additional neural resources to effectively cope with an emotion-eliciting event is provided by

501 a meta-analysis [39]. This analysis shows that ventromedial prefrontal cortex activation is present in

502 diverse, but related experimental domains such as fear extinction, cognitive emotion regulation

503 (reappraisal), and placebo-mediated changes in negative affect. Only during reappraisal and placebo-

504 mediated changes, however, insular and cingulate regions were activated as well, indicating that these

505 two regions specifically support more complex functions as compared to basic extinction processes,

506 for which less distributed activation, primarily of the ventromedial prefrontal cortex, seems to be

507 sufficient.

A third notable result is that a principled approach to experimental design and data analysis

509 allows for further insights beyond the canonical results. On the one hand, as detailed below, we were

510 able to identify different temporal response profiles for the sources and targets of emotional

511 regulation. On the other hand, we were also able to differentiate between main and interaction effects

512 for the picture and regulation conditions.

The first insight regards a dissociation between transient/sustained responses and the

514 targets/sources of emotional regulation. During the stimulation phase, but not necessarily during the

515 post-stimulation phase, we observed a primarily transient response pattern within the amygdala,

516 considering the statistical significance, spatial extent, and consistency across hemispheres. This

517 pattern is consistent with its presumed alerting function [40]. In that sense, the amygdala initiates a

518 response, but its activation does not represent the emotional response per se, which also includes

519 down-stream activation in for example motor systems [41]. As long as the environment does not

520 change any further, as it is the case for static pictures, continued activation of such an alerting structure

521 is not needed, and hence the transient response. These results are compatible with Paret, Kluetsch, 
This is a preprint of a revised manuscript submitted to PlosOne

522 Ruf, Demirakca, Kalisch, Schmahl, et al. (42), who found that primarily the anterior insula, but also

523 the left amygdala, exhibits a transient response pattern in event-related designs with longer picture

524 durations. Both studies underscore that results crucially depend on the analytical model, and that

525 modeling choices must be made consciously, based on a priori evidence.

\section{Concurrent emotion regulation effects in different picture conditions}

Another insight concerns the question whether or not emotion regulation is a domain-general

528 or a domain-specific process; that is, whether regulation strategies and their effectiveness generalize

529 across stimulus characteristics. Morawetz, Bode, Derntl and Heekeren (43) already showed, that

530 activation of brain areas during emotion regulation generalizes across stimulus categories (IAPS

531 pictures versus faces, film clips, reward, pain, scripts, and shapes). Our results point to a

532 generalization across stimulus characteristics (in this case: valence), which is also in line with

533 domain-general views on the brain basis of emotional reactivity [44]. The observation of decreased

534 amygdala activation for neutral pictures during the distancing condition as compared to the permit

535 condition may indicate that the distinction between negative and neutral pictures is a gradual one: we

536 speculate that the mere presentation of (bright) pictures in the dark environment of the scanner elicits

537 an unspecific alerting/arousing response - and that this response can be the target of emotional

538 regulation.

539 Although, in our experiment, the difference between distance and permit was greater for

540 negative than neutral pictures, the interaction effect between picture and regulation was relatively

541 small, for example with regard to its spatial extent, and not as consistent as for the main effects.

542 Similar results were also reported by Walter and colleagues [9] with a similar experimental design,

543 indicating that there is a minor (quantitative), but no fundamental (qualitative) difference between

544 distancing oneself from a negative or a neutral stimulus. The overall activation patterns of the neutral 
This is a preprint of a revised manuscript submitted to PlosOne

545 and negative pictures and the joint analysis are also similar in that they all lead to activation of the

546 right middle frontal gyrus and inferior parietal lobe. This supports the idea of a core network

547 consisting of middle frontal and inferior parietal regions involved in general regulation efforts, and

548 that additional components are added to this network depending on the particular task at hand. From

549 a conceptual point of view, we suggest that it is therefore not just either domain-general or just

550 domain-specific processing that characterizes emotion regulation, but both - depending on the 551 particular region.

\section{Effects of time}

The second major focus of our study was on the temporal aspects of emotion regulation. We implemented a slow event-related design to achieve a balance between stimulation (timepoint 1) and post-stimulation (timepoint 2) periods, and also investigated the response to previously regulated and non-regulated pictures after short (10 min, timepoint 3) and long (one week, timepoint 4) intervals.

\section{Immediate emotion regulation aftereffects at timepoint 2}

We observed paradoxically increased amygdala activation after regulation at timepoint 2 .

559 However, these did not appear as a fully crossed interaction and were dependent on the statistical activation model: In the voxel-based analysis, we found greater activation during distance than during permit in the post-stimulation phase only for sustained responses and interaction effects between time and regulation for both the transient and sustained responses. These results can be regarded as partial replications of the paradoxical rebound effect reported by Walter and colleagues [9]. They confirm the original proposition of post-stimulation activation differences across conditions as well as activation increases and decreases within conditions, but disagree with respect to the exact nature of this effect. For example, the original effect was found using the activation obtained from extracted 
This is a preprint of a revised manuscript submitted to PlosOne

568 how the amygdala responses were modeled, and on the other hand, they did not necessarily occur at

569 precisely the same locations (in terms of MNI coordinates), although all of them occurred within the

570 amygdala ROI. Although this implies that a functional interpretation of this effect needs to be made

571 in a more careful way, both studies support the notion of an interdependence of stimulation and post-

572 stimulation periods. One could argue that the amount of cortical engagement during reappraisal is

573 reduced as a function of time spent on implementing a certain reappraisal strategy [45]. This in turn

574 might lead to an increase of amygdala activation after initial down-regulation.

Previous studies proposed that the concept of emotional aftereffects be extended to other

regions apart from the amygdala, and to other types of interaction besides paradoxical, or crossed,

ones. In this regard, a key result of Lamke and colleagues [22] is that reverse task-rest interactions

(decreased activation during regulation but increased activation during relaxation/rest) were present

in the amygdala after emotion down-regulation, whereas concordant task-rest interactions (increased

activation during both regulation and relaxation/rest periods) were apparent in the prefrontal cortex.

Comparable results were obtained in our study. We also observed task(stimulation)-rest(poststimulation) interactions for negative pictures in regions beyond the amygdala, for example in the occipital cortex and the ventromedial frontal / subgenual cingulate cortex.

\section{4}

\section{Short- and long-term emotion regulation effects (timepoints 3 and 4)}

In our study, previous regulation had also an impact on amygdala activation during the reexposure sessions: both after $10 \mathrm{~min}$ and after 1 week: Previously down-regulated items showed greater activation within the left and right amygdala than previously non-regulated items. This effect was, however, not limited to negative pictures, but also appeared for neutral ones. These findings differ from previous results $[9,24]$ indicating that emotion regulation effects persisted over time, that is that previously down-regulated items were associated with decreased amygdala activation. 
This is a preprint of a revised manuscript submitted to PlosOne

591 Hermann and colleagues [46] also investigated re-exposure to previously (one week) regulated

592 stimuli and found not difference between previously distanced and permitted stimuli in amygdala

593 activation nor in ratings of emotional experience. However, the authors reported an effect of previous

594 reinterpretation, another reappraisal tactic, of negative stimuli leading to increased amygdala

595 activation and reduced emotional experience during re-exposure. Additionally, there was no

596 difference in re-exposure effects between reinterpretation and distancing. Since we did not record

597 arousal ratings during re-exposure, we cannot conclude that being confronted with negative situations

598 that have been previously regulated via distancing leads to reduced emotional experience.

599 Nevertheless, the findings by Hermann and colleagues [46] might suggest, that this is a possible 600 consequence of reappraisal, albeit at least for reinterpretation.

All of the aforementioned studies show that regulation effects persist over time, albeit in

602 different ways. Although it has previously been shown that re-exposure effects also depend on the 603 regulation strategy [47], this is no explanation for the specific pattern of results in our study, and why 604 there are differences with regard to the Walter et al. study, given the similarity of the experimental 605 setup. This remains an open question and needs to be the subject of further replication efforts.

\section{Limitations}

We will now address the limitations of our study, which primarily concern the experimental design and the generality of our results. First, for reasons of design efficiency we only investigated negative vs. neutral pictures. This did not allow us to disentangle the impact of different levels of

610 arousal and valence, since negative and neutral pictures simultaneously differ in both dimensions. It

611 has been shown, though, that this is a relevant distinction with regard to amygdala [48] and prefrontal 612 activation [49]. Including positive pictures would have allowed for a more comprehensive 613 investigation in this regard. Further, a comparison of negative and neutral pictures alone does not 
This is a preprint of a revised manuscript submitted to PlosOne

614 allow conclusions about different kinds of emotion, if a discrete model of emotions is assumed. Again,

615 there is evidence that different negative emotions such as fear, anxiety, sadness, and anger, and also

616 positive vs. negative emotions are differentially susceptible to emotional regulation [50-52].

Second, this experiment was not designed to investigate the effects of different reappraisal

tactics. While there is a common core network of emotion regulation, which we also found in our

619 study, this does not necessarily mean that our results hold for different regulation tactics or even other

620 strategies. Similar to the inclusion of further stimulus categories, the inclusion of further strategies

621 would have been desirable, but would have necessitated the presentation of trials in a more rapid

622 succession, which conflicted with our decision for a slow event-related experimental design. In that

623 sense, there is a trade-off between design choices that cannot be resolved within a single study.

Third, the interpretation of our results rests on the assumption that participants on average

could successfully implement the distance and permit instructions. Although we took effort to control

for this by means of detailed instructions, training, and subsequent self-reports, it is possible that alternative strategies, involuntarily regulation, or mind-wandering were applied by the participants.

628 We assume, though, that the impact of such regulation variants is random and just increases error variance in the data. However, to investigate whether such variability can lead to any systematic bias,

630 further targeted studies are needed, e.g. to distinguish voluntary vs. involuntary emotion regulation.

Fourth, the interpretation of results for the re-exposure session is impacted by the drop-out of

632 participants, which led to a reduced sample size for this part of the experiment. Also, additional

633 arousal ratings during this phase could have provided additional information on the short- and long-

634 term effects of emotion regulation. Finally, more variables than those considered for the present study

635 play a role in emotion regulation: on the one hand, variation among individuals, be it clinical or non-

636 clinical, will impact cognitive emotion regulation in a sense that different strategies will have different 
This is a preprint of a revised manuscript submitted to PlosOne

637 efficacies for different individuals. This could, for example, take the form of an association of 638 successful reappraisal with less trait anxiety and more positive daily emotion as well greater 639 activation in medial and lateral prefrontal regions [53]. On the other hand, successful vs. deficient 640 emotion regulation will lead, in a developmental perspective, to different clinical trajectories; for 641 example, emotion regulations skills predict the severity of anxiety symptoms across an interval of 5

642 years [54], and improving emotion regulation skills has been shown to enhance the efficacy of 643 psychotherapeutic interventions [55]. Emotion regulation deficits are present in almost all clinical 644 psychological disorders, which in turn are characterized by differential strategies of unsuccessful 645 emotion regulation. Especially in the clinical context, comparing short term beneficial vs long term 646 dysfunctional emotion regulation attempts appears very promising. To extend the study design to 647 other populations than young and healthy students is therefore a necessary step for translating emotion 648 regulation research into application contexts.

\section{Conclusions}

Many of the issues central to this study have already been investigated, but not necessarily in a single study, and indeed, often in isolation. Further integration within the field requires some "firm ground", that is canonical results of established and replicable findings that can be derived from systematic reviews and quantitative meta-analyses, but also from replication efforts such as the present study. A key motivation of our study was to replicate and refine some core and emerging results in cognitive emotion regulation. Its main contribution is the joint and coherent consideration of a subset of relevant experimental factors - emotional processing and regulation as well as their temporal trajectories - and their effects on the activation patterns of known emotion generating and

658 regulating networks. Our results confirm and extend previous characterizations of these networks, 659 but also call for further investigation especially of their temporal dynamics. 


\section{Acknowledgments}

661 We thank Patricia Schimm for her great support with providing Open Data and Open 662 Materials.

\section{Data Availability Statement}

664 Data and materials are provided at the Open Science Framework (https://osf.io/mg5ac/).

665 Specifically, we provide data for first- and second-level fMRI analyses, thee respective SPM code,

666 ROI masks, behavioral data (arousal ratings, age, sex), the emotion regulation experiment, and R-

667 code for reproducing the analyses and results. We are not able to provide raw and preprocessed fMRI

668 data due to data privacy reasons.

669 


\section{References}

671 1. Etkin A, Buchel C, Gross JJ. The neural bases of emotion regulation. Nat Rev Neurosci. 2015;16(11):693-700. doi: 10.1038/nrn4044. PubMed PMID: 26481098.

2. Gross JJ. Antecedent- and response-focused emotion regulation: divergent consequences for experience, expression, and physiology. J Pers Soc Psychol. 1998;74(1):224-37. PubMed PMID: 9457784.

3. Powers JP, LaBar KS. Regulating emotion through distancing: A taxonomy, neurocognitive model, and supporting meta-analysis. Neurosci Biobehav Rev. 2019;96:155-73. doi: 10.1016/j.neubiorev.2018.04.023. PubMed PMID: 30502352; PubMed Central PMCID: PMCPMC6326885.

4. Webb TL, Miles E, Sheeran P. Dealing with feeling: a meta-analysis of the effectiveness of strategies derived from the process model of emotion regulation. Psychol Bull. 2012;138(4):775-808. doi: 10.1037/a0027600. PubMed PMID: 22582737.

5. Aldao A, Nolen-Hoeksema S, Schweizer S. Emotion-regulation strategies across psychopathology: A meta-analytic review. Clin Psychol Rev. 2010;30(2):217-37. doi: 10.1016/j.cpr.2009.11.004. PubMed PMID: 20015584.

6. Gross JJ. Emotion Regulation: Conceptual and Empirical Foundations. In: Gross JJ, editor. Handbook of Emotion Regulation. 2. New York London: The Guilford Press; 2014. p. 3-20. Psychophysiology. 2002;39(3):281-91. doi: 10.1017.S0048577201393198. PubMed PMID: 12212647. 
This is a preprint of a revised manuscript submitted to PlosOne

8. Ochsner KN, Bunge SA, Gross JJ, Gabrieli JD. Rethinking feelings: an FMRI study of the cognitive regulation of emotion. Journal of Cognitive Neuroscience. 2002;14(8):1215-29. doi: 10.1162/089892902760807212. PubMed PMID: 12495527.

9. Walter H, von Kalckreuth A, Schardt D, Stephan A, Goschke T, Erk S. The temporal dynamics of voluntary emotion regulation. PloS One. 2009;4(8):e6726. doi: 10.1371/journal.pone.0006726. PubMed PMID: 21949675; PubMed Central PMCID: PMCPMC3175755.

10. Dörfel D, Lamke JP, Hummel F, Wagner U, Erk S, Walter H. Common and differential neural networks of emotion regulation by Detachment, Reinterpretation, Distraction, and Expressive Suppression: a comparative fMRI investigation. NeuroImage. 2014;101:298-309. doi: 10.1016/j.neuroimage.2014.06.051. PubMed PMID: 24993897.

11. Kalisch R, Wiech K, Critchley HD, Seymour B, O'Doherty JP, Oakley Da, et al. Anxiety reduction through detachment: subjective, physiological, and neural effects. Journal of cognitive neuroscience. 2005;17(6):874-83. doi: 10.1162/0898929054021184. PubMed PMID: 15969906.

12. Buhle JT, Silvers JA, Wager TD, Lopez R, Onyemekwu C, Kober H, et al. Cognitive reappraisal of emotion: a meta-analysis of human neuroimaging studies. Cerebral Cortex. 2014;24(11):2981-90. doi: 10.1093/cercor/bht154. PubMed PMID: 23765157; PubMed Central PMCID: PMCPMC4193464.

13. Ochsner KN, Gross JJ. The cognitive control of emotion. Trends in Cognitive Sciences. 2005;9(5):242-9. doi: 10.1016/j.tics.2005.03.010. PubMed PMID: 15866151.

14. Frank DW, Dewitt M, Hudgens-Haney M, Schaeffer DJ, Ball BH, Schwarz NF, et al. Emotion regulation: quantitative meta-analysis of functional activation and deactivation. Neuroscience 
This is a preprint of a revised manuscript submitted to PlosOne

and Biobehavioral Reviews. 2014;45:202-11. doi: 10.1016/j.neubiorev.2014.06.010. PubMed PMID: 24984244.

15. Kohn N, Eickhoff SB, Scheller M, Laird AR, Fox PT, Habel U. Neural network of cognitive emotion regulation--an ALE meta-analysis and MACM analysis. NeuroImage. 2014;87:34555. doi: 10.1016/j.neuroimage.2013.11.001. PubMed PMID: 24220041; PubMed Central PMCID: PMCPMC4801480.

16. Banks SJ, Eddy KT, Angstadt M, Nathan PJ, Phan KL. Amygdala-frontal connectivity during emotion regulation. Social cognitive and affective neuroscience. 2007;2(4):303-12. doi: 10.1093/scan/nsm029. PubMed PMID: 18985136; PubMed Central PMCID: PMCPMC2566753.

17. Morawetz C, Bode S, Baudewig J, Heekeren HR. Effective amygdala-prefrontal connectivity predicts individual differences in successful emotion regulation. Social Cognitive and Affective Neuroscience 2017;12(4):569-85. Epub 2016/12/22. doi: 10.1093/scan/nsw169. PubMed PMID: 27998996; PubMed Central PMCID: PMCPMC5390747.

18. Dörfel D, Gartner A, Scheffel C. Resting State Cortico-Limbic Functional Connectivity and Dispositional Use of Emotion Regulation Strategies: A Replication and Extension Study. Front Behav Neurosci. 2020;14:128. doi: 10.3389/fnbeh.2020.00128. PubMed PMID: 32848654; PubMed Central PMCID: PMCPMC7399345.

19. Golkar A, Lonsdorf TB, Olsson A, Lindstrom KM, Berrebi J, Fransson P, et al. Distinct contributions of the dorsolateral prefrontal and orbitofrontal cortex during emotion regulation. PloS One. 2012;7(11):e48107. doi: 10.1371/journal.pone.0048107. PubMed PMID: 23144849 ; PubMed Central PMCID: PMCPMC3492343. 
This is a preprint of a revised manuscript submitted to PlosOne

20. Kanske P, Heissler J, Schonfelder S, Bongers A, Wessa M. How to regulate emotion? Neural networks for reappraisal and distraction. Cerebral Cortex. 2011;21(6):1379-88. doi: 10.1093/cercor/bhq216. PubMed PMID: 21041200.

21. Langner R, Leiberg S, Hoffstaedter F, Eickhoff SB. Towards a human self-regulation system: Common and distinct neural signatures of emotional and behavioural control. Neuroscience and Biobehavioral Reviews. 2018;90:400-10. doi: 10.1016/j.neubiorev.2018.04.022. PubMed PMID: 29730485; PubMed Central PMCID: PMCPMC5994341.

22. Lamke JP, Daniels JK, Dörfel D, Gaebler M, Abdel Rahman R, Hummel F, et al. The impact of stimulus valence and emotion regulation on sustained brain activation: task-rest switching in emotion. PloS One. 2014;9(3):e93098. doi: 10.1371/journal.pone.0093098. PubMed PMID: 24682003; PubMed Central PMCID: PMCPMC3969367.

23. Hermann A, Kress L, Stark R. Neural correlates of immediate and prolonged effects of cognitive reappraisal and distraction on emotional experience. Brain Imaging Behav. 2017;11(5):1227-37. doi: 10.1007/s11682-016-9603-9. PubMed PMID: 27709512.

24. Denny BT, Inhoff MC, Zerubavel N, Davachi L, Ochsner KN. Getting Over It: Long-Lasting Effects of Emotion Regulation on Amygdala Response. Psychological Science. 2015;26(9):1377-88. doi: 10.1177/0956797615578863. PubMed PMID: 26231911; PubMed Central PMCID: PMCPMC4567486.

25. Erk S, Mikschl A, Stier S, Ciaramidaro A, Gapp V, Weber B, et al. Acute and sustained effects of cognitive emotion regulation in major depression. J Neurosci. 2010;30(47):15726-34. doi: 30/47/15726 [pii] 10.1523/JNEUROSCI.1856-10.2010. PubMed PMID: 21106812.

26. Gärtner A, Dörfel D, Diers K, Witt SH, Strobel A, Brocke B. Impact of FAAH genetic variation on fronto-amygdala function during emotional processing. European archives of psychiatry 
This is a preprint of a revised manuscript submitted to PlosOne

and clinical neuroscience. 2019;269(2):209-21. doi: 10.1007/s00406-018-0944-9. PubMed PMID: 30291441.

27. Scheffel C, Diers K, Schonfeld S, Brocke B, Strobel A, Dörfel D. Cognitive emotion regulation and personality: an analysis of individual differences in the neural and behavioral correlates of successful reappraisal. Personal Neurosci. 2019;2:e11. doi: 10.1017/pen.2019.11. PubMed PMID: 32435746; PubMed Central PMCID: PMCPMC7219681.

28. Simmons JP, Nelson LD, Simonsohn U. A 21 Word Solution. Dialogue - The Official Newsletter of the Society for Personality and Social Psychology. 2012;26(2):4-7.

29. Diers K, Weber F, Brocke B, Strobel A, Schonfeld S. Instructions matter: a comparison of baseline conditions for cognitive emotion regulation paradigms. Frontiers in Psychology 2014;5:347. doi: 10.3389/fpsyg.2014.00347. PubMed PMID: 24808872; PubMed Central PMCID: PMCPMC4009445.

30. Lang PJ, Bradley MM, Cuthbert BN. International Affective Picture System (IAPS): Technical manual and affective ratings: NIMH Center for the Study of Emotion and Attention; 1997.

31. Wessa M, Kanske P, Neumeister P, Bode K, Heissler J, Schönfelder S. EmoPics: Subjektive und psychophysiologische Evaluation neuen Bildmaterials für die klinisch-biopsychologische Forschung. Zeitschrift für Klinische Psychologie und Psychotherapie 2010;39(Suppl. 1/11):77.

32. Wickham H. ggplot2: Elegant Graphics for Data Analysis. New York2009.

33. Eickhoff SB, Stephan KE, Mohlberg H, Grefkes C, Fink GR, Amunts K, et al. A new SPM toolbox for combining probabilistic cytoarchitectonic maps and functional imaging data. NeuroImage. 2005;25(4):1325-35. doi: 10.1016/j.neuroimage.2004.12.034. PubMed PMID: 15850749. 
This is a preprint of a revised manuscript submitted to PlosOne

34. Paschke LM, Dörfel D, Steimke R, Trempler I, Magrabi A, Ludwig VU, et al. Individual differences in self-reported self-control predict successful emotion regulation. Social Cognitive and Affective Neuroscience. 2016;11(8):1193-204. doi: 10.1093/scan/nsw036. PubMed PMID: 27013102; PubMed Central PMCID: PMCPMC4967798.

35. Klumpp H, Bhaumik R, Kinney KL, Fitzgerald JM. Principal component analysis and neural predictors of emotion regulation. Behav Brain Res. 2018;338:128-33. doi: 10.1016/j.bbr.2017.10.024. PubMed PMID: 29061386; PubMed Central PMCID: PMCPMC5815323.

36. Morawetz C, Kellermann T, Kogler L, Radke S, Blechert J, Derntl B. Intrinsic functional connectivity underlying successful emotion regulation of angry faces. Social Cognitive and Affective Neuroscience. 2016;11(12):1980-91. doi: 10.1093/scan/nsw107. PubMed PMID: 27510495 ; PubMed Central PMCID: PMCPMC5141959.

37. Messina I, Bianco S, Sambin M, Viviani R. Executive and semantic processes in reappraisal of negative stimuli: insights from a meta-analysis of neuroimaging studies. Frontiers in psychology. 2015;6:956. doi: 10.3389/fpsyg.2015.00956. PubMed PMID: 26217277; PubMed Central PMCID: PMCPMC4499672.

38. Geva S, Jones PS, Crinion JT, Price CJ, Baron JC, Warburton EA. The neural correlates of inner speech defined by voxel-based lesion-symptom mapping. Brain. 2011;134(Pt 10):307182. Epub 2011/10/07. doi: 10.1093/brain/awr232. PubMed PMID: 21975590; PubMed Central PMCID: PMCPMC3187541.

39. Diekhof EK, Geier K, Falkai P, Gruber O. Fear is only as deep as the mind allows: a coordinate-based meta-analysis of neuroimaging studies on the regulation of negative affect. NeuroImage. 2011;58(1):275-85. doi: 10.1016/j.neuroimage.2011.05.073. PubMed PMID: 21669291. 
This is a preprint of a revised manuscript submitted to PlosOne

40. Liddell BJ, Brown KJ, Kemp AH, Barton MJ, Das P, Peduto A, et al. A direct brainstemamygdala-cortical 'alarm' system for subliminal signals of fear. NeuroImage. 2005;24(1):23543. doi: 10.1016/j.neuroimage.2004.08.016. PubMed PMID: 15588615.

41. Grezes J, Valabregue R, Gholipour B, Chevallier C. A direct amygdala-motor pathway for emotional displays to influence action: A diffusion tensor imaging study. Human brain mapping. 2014;35(12):5974-83. doi: 10.1002/hbm.22598. PubMed PMID: 25053375; PubMed Central PMCID: PMCPMC6869045.

42. Paret C, Kluetsch R, Ruf M, Demirakca T, Kalisch R, Schmahl C, et al. Transient and sustained BOLD signal time courses affect the detection of emotion-related brain activation in fMRI. NeuroImage. 2014;103:522-32. Epub 2014/09/11. doi: 10.1016/j.neuroimage.2014.08.054. PubMed PMID: 25204866.

43. Morawetz C, Bode S, Derntl B, Heekeren HR. The effect of strategies, goals and stimulus material on the neural mechanisms of emotion regulation: A meta-analysis of fMRI studies. Neurosci Biobehav Rev. 2017;72:111-28. doi: 10.1016/j.neubiorev.2016.11.014. PubMed PMID: 27894828.

44. Lindquist KA, Wager TD, Kober H, Bliss-Moreau E, Barrett LF. The brain basis of emotion: a meta-analytic review. Behavioral and Brain Sciences. 2012;35(3):121-43. doi: 10.1017/S0140525X11000446. PubMed PMID: 22617651; PubMed Central PMCID: PMCPMC4329228.

45. Sarkheil P, Klasen M, Schneider F, Goebel R, Mathiak K. Amygdala response and functional connectivity during cognitive emotion regulation of aversive image sequences. European archives of psychiatry and clinical neuroscience. 2019;269(7):803-11. doi: 10.1007/s00406018-0920-4. PubMed PMID: 30008118. 
This is a preprint of a revised manuscript submitted to PlosOne

829 46. Hermann A, Neudert MK, Schafer A, Zehtner RI, Fricke S, Seinsche RJ, et al. Lasting Effects Of Cognitive Emotion Regulation: Neural Correlates Of Reinterpretation And Distancing. Soc Cogn Affect Neurosci. 2020. doi: 10.1093/scan/nsaa159. PubMed PMID: 33227135.

47. Thiruchselvam R, Blechert J, Sheppes G, Rydstrom A, Gross JJ. The temporal dynamics of emotion regulation: an EEG study of distraction and reappraisal. Biological psychology. 2011;87(1):84-92. doi: 10.1016/j.biopsycho.2011.02.009. PubMed PMID: 21354262.

48. Zald DH. The human amygdala and the emotional evaluation of sensory stimuli. Brain Res

49. Grimm S, Schmidt CF, Bermpohl F, Heinzel A, Dahlem Y, Wyss M, et al. Segregated neural representation of distinct emotion dimensions in the prefrontal cortex-an fMRI study. NeuroImage. 2006;30(1):325-40. doi: 10.1016/j.neuroimage.2005.09.006. PubMed PMID: 16230029.

50. Mak AK, Hu ZG, Zhang JX, Xiao ZW, Lee TM. Neural correlates of regulation of positive and negative emotions: an fmri study. Neuroscience letters. 2009;457(2):101-6. doi: 10.1016/j.neulet.2009.03.094. PubMed PMID: 19429172.

51. Wheelock MD, Sreenivasan KR, Wood KH, Ver Hoef LW, Deshpande G, Knight DC. Threatrelated learning relies on distinct dorsal prefrontal cortex network connectivity. NeuroImage. 2014;102 Pt 2:904-12. doi: 10.1016/j.neuroimage.2014.08.005. PubMed PMID: 25111474; PubMed Central PMCID: PMCPMC4252829.

52. Yoshimura S, Okamoto Y, Yoshino A, Kobayakawa M, Machino A, Yamawaki S. Neural basis of anticipatory anxiety reappraisals. PloS One. 2014;9(7):e102836. doi: 10.1371/journal.pone.0102836. PubMed PMID: 25048028; PubMed Central PMCID: PMC4105563. 
This is a preprint of a revised manuscript submitted to PlosOne

852 53. Uchida M, Biederman J, Gabrieli JD, Micco J, de Los Angeles C, Brown A, et al. Emotion regulation ability varies in relation to intrinsic functional brain architecture. Social Cognitive and Affective Neuroscience. 2015;10(12):1738-48. doi: 10.1093/scan/nsv059. PubMed PMID: 25999363; PubMed Central PMCID: PMCPMC4666109.

856 54. Wirtz CM, Hofmann SG, Riper H, Berking M. Emotion regulation predicts anxiety over a five-year interval: a cross-lagged panel analysis. Depress Anxiety. 2014;31(1):87-95. doi: 10.1002/da.22198. PubMed PMID: 24151095.

859 55. Berking M, Wupperman P, Reichardt A, Pejic T, Dippel A, Znoj H. Emotion-regulation skills 860 as a treatment target in psychotherapy. Behaviour research and therapy. 2008;46(11):1230-7. 861 doi: 10.1016/j.brat.2008.08.005. PubMed PMID: 18835479. 